\title{
DDA CALCULATION OF LIGHT SCATTERING BY BIREFRINGENT AND POLYCRYSTALLINE SUBMICRON SPHERES
}

\author{
Imon Kalyan ${ }^{1}$, C. V. Krishnamurthy ${ }^{2}$ \\ ${ }^{1}$ Department of Physics, Indian Institute of Technology Madras, Chennai-600036, India \\ ${ }^{2}$ Department of Physics, Indian Institute of Technology Madras, Chennai-600036, India
}

\begin{abstract}
Structure, morphology and refractive index play an important role in light scattering by small particles. The effect of internal anisotropy of a particle in light scattering is the interest of our study. Scattering cross section is calculated for spheres of anatase and rutile phases of titanium dioxide, taking account of its internal anisotropy using discrete dipole approximation. Scattering spectra obtained for the birefringent single and polycrystalline sphere are compared to that obtained for an isotropic sphere with weighted averaged refractive index.
\end{abstract}

\section{INTRODUCTION}

Micro and nano-particles of certain dielectric materials have been found very useful in diverse fields of optics and photonics because of their unique interaction with light. Dielectric particles of spherical and cylindrical shape show morphology dependent resonance modes in its scattering spectrum [1]. Such modes have been investigated for their applications as sensors, filters etc. [2] as they are very sensitive to size, shape and refractive index of the particle. However, several dielectric materials are birefringent in nature and also particles of dielectric material in nano or micron size could be polycrystalline. Such internal anisotropy may result in changes of the scattering spectrum. The internal anisotropy and polycrystallinity leads to rich spectral characteristics with potential applications.

Numerical investigations have been very important in predicting the interaction of electromagnetic waves with small particles. Mie theory has been well developed to explain the scattering and absorption of light by homogeneous spheres [3], but is not suitable to deal with particles with arbitrary shape and birefringence. Discrete dipole approximation (DDA) is a well-known numerical method to handle such particles $[4,5]$.

Titanium dioxide $\left(\mathrm{TiO}_{2}\right)$ is a birefringent material, which has been found useful in various fields like pigmentation, photo catalysis, solar cells, sensing etc. [6-8]. $\mathrm{TiO}_{2}$ spheres have been reported to show morphology dependent resonance peaks in its scattering spectrum while interacted with light [9]. Due to such behaviour $\mathrm{TiO}_{2}$ spheres have also been found applicable in template-guided self-assembly structures with optoelectronic applications [8]. Rutile and anatase phases of $\mathrm{TiO}_{2}$ are acclaimed most important from an application point of view. Birefringence is present in both of these phases. While rutile shows positive uniaxial structure, anatase shows negative uniaxial structure. Refractive index and birefringence of rutile phased $\mathrm{TiO}_{2}$ are significantly high compared to most of the dielectric materials. Birefringence of anatase is 0.099 while that of rutile is 0.32 at the wavelength of $500 \mathrm{~nm}$.
In this study we are presenting numerical calculations of scattering spectrum of birefringent $\mathrm{TiO}_{2}$ sphere with single as well as many crystal domains using discrete dipole approximation (DDA). Effect of birefringence and polycrystallinity on light scattering has been studied for both rutile and anatase phases with different numbers of crystal domain.

\section{METHOD OF CALCULATION}

In DDA calculations a particle is considered to be made of periodic arrays of discrete polarizable entities with the distance between two entities $d$ satisfying the following relation-

$$
|m| k d<1
$$

Where $m$ is the refractive index of the material and $k$ is the wave number of the incident light. Each entity at $i^{\text {th }}$ position is assigned a polarizability tensor . Dipole moment, associated with each polarizable entity is induced by the incident field and the fields at location $i$ due to all the other induced dipoles.

$$
\vec{p}_{i}=\alpha_{i}\left(\vec{E}_{i n c, i}-\sum_{j \neq i} \vec{A}_{i, j} \vec{p}_{j}\right)
$$

Where, $-\vec{A}_{i, j} \vec{p}_{j}$ is the electric field at position $i$ due to the dipole at $j$.

$$
\vec{A}_{i i}=\alpha_{i}^{-1}, \quad i=j
$$




$$
\vec{A}_{i j} \vec{p}_{j}=\frac{\exp \left(-i k r_{i j}\right)}{r_{i j}^{3}}\left[k^{2} \vec{r}_{i j} \times\left(\vec{r}_{i j} \times \vec{p}_{j}\right)+\frac{\left(1-i k r_{i j}\right)}{r_{i j}^{2}} \times\left\{r_{i j}^{2} \vec{p}_{j}-3 \vec{r}_{i j}\left(\vec{r}_{i j} \cdot \vec{p}_{j}\right)\right\}\right], \quad i \neq j
$$

$\vec{p}_{i}$ at each $\mathrm{i}^{\text {th }}$ position can be calculated solving equation (2) with iterative techniques. Absorption cross section, scattering cross section etc. can be calculated from the value of dipole moment of each polarizable entity as given in equation (3) and (4).

Absorption cross section:

$$
C_{a b s}=\frac{4 \pi k}{\left|E_{i n c}\right|^{2}} \sum_{i=1}^{N}\left\{\operatorname{Im}\left[\vec{p}_{i} \cdot\left(\alpha_{i}^{-1}\right)^{*} \vec{p}_{i}^{*}\right]-\frac{2}{3} k^{3} \vec{p}_{i} \cdot \vec{p}_{i}^{*}\right\}
$$

Scattering cross section:

$$
C_{\text {sca }}=\frac{k^{4}}{\left|E_{\text {inc }}\right|^{2}} \int d \Omega\left|\sum_{i=1}^{N}\left[\vec{p}_{i}-\hat{n}\left\{\hat{n}_{\cdot} \cdot \vec{p}_{i}\right\}\right] \exp \left\{-i k \hat{n} \cdot \vec{r}_{i}\right\}\right|^{2}
$$

where $\hat{n}$ is a unit vector in the direction of scattering and $d \Omega$ is the element of solid angle.

Sharp peaks are observed in the scattering spectrum of dielectric micro spheres which are better known as morphology dependent resonance modes [1]. Spectral positions of these modes can be found by solving Maxwell's equations in spherical polar coordinates applying boundary conditions. The mode solutions are classified as transverse magnetic (TM) and transverse electric (TE) modes depending on the alignment of the magnetic and electric field transverse to the radial direction. The solutions to Maxwell's equations for a sphere of radius $a$ lead to the following condition for a mode [10]

$$
P \frac{j_{l}^{\prime}(m x)}{j_{l}(m x)}=\frac{n_{l}^{\prime}(x)}{n_{l}(x)}
$$

Where $\dot{j}_{l}$ and $n_{l}$ are spherical Bessel and Neumann functions respectively and the prime indicates the derivative with respect to their arguments. $l$ is the angular momentum mode number, $x=k a$ and $P=m$ for TE mode and $P=1 / m$ for TM mode.

For the calculation of scattering spectrum using DDA, a sphere of diameter $500 \mathrm{~nm}$ is constructed by cubically arranged polarizable entities. Number of entities is chosen such that the spacing between the dipoles $d$ follows equation (1) and the pattern follows that obtained from Mie calculation when calculated for an isotropic sphere with refractive index equal to the highest of the ordinary $\left(\mathrm{n}_{\mathrm{o}}\right)$ and extraordinary $\left(\mathrm{n}_{\mathrm{e}}\right)$ refractive indices. The spheres for anatase phase are made of about $2.68 \times 10^{5}$ numbers of polarizable entities. In the case of rutile sphere, scattering spectrum is divided into two ranges due to its high refractive index in the lower wavelength region. For wavelength below $600 \mathrm{~nm}$, spheres are represented by about $10^{6}$ and above $600 \mathrm{~nm}$, spheres are represented by about $5 \times 10^{5}$ numbers of polarizable entities. Each polarizable entity is represented by a polarizability tensor whose values are obtained from the bulk values of refractive indices $[11,12]$. DDA calculations have been performed using DDSCAT codes $[5,6]$.

In the case of birefringent single crystalline sphere (BSCS) the polarizability tensor assigned is considered to be diagonal in the lab frame, with its extra-ordinary refractive index along $\mathrm{z}$ axis as shown in figure 1(a). A plane polarized light is incident on the particle from $x$ direction (perpendicular to principal axis) as well as from $\mathrm{z}$ direction (along principal axis). Scattering cross section is calculated for linearly polarized light for both parallel and perpendicular components. 

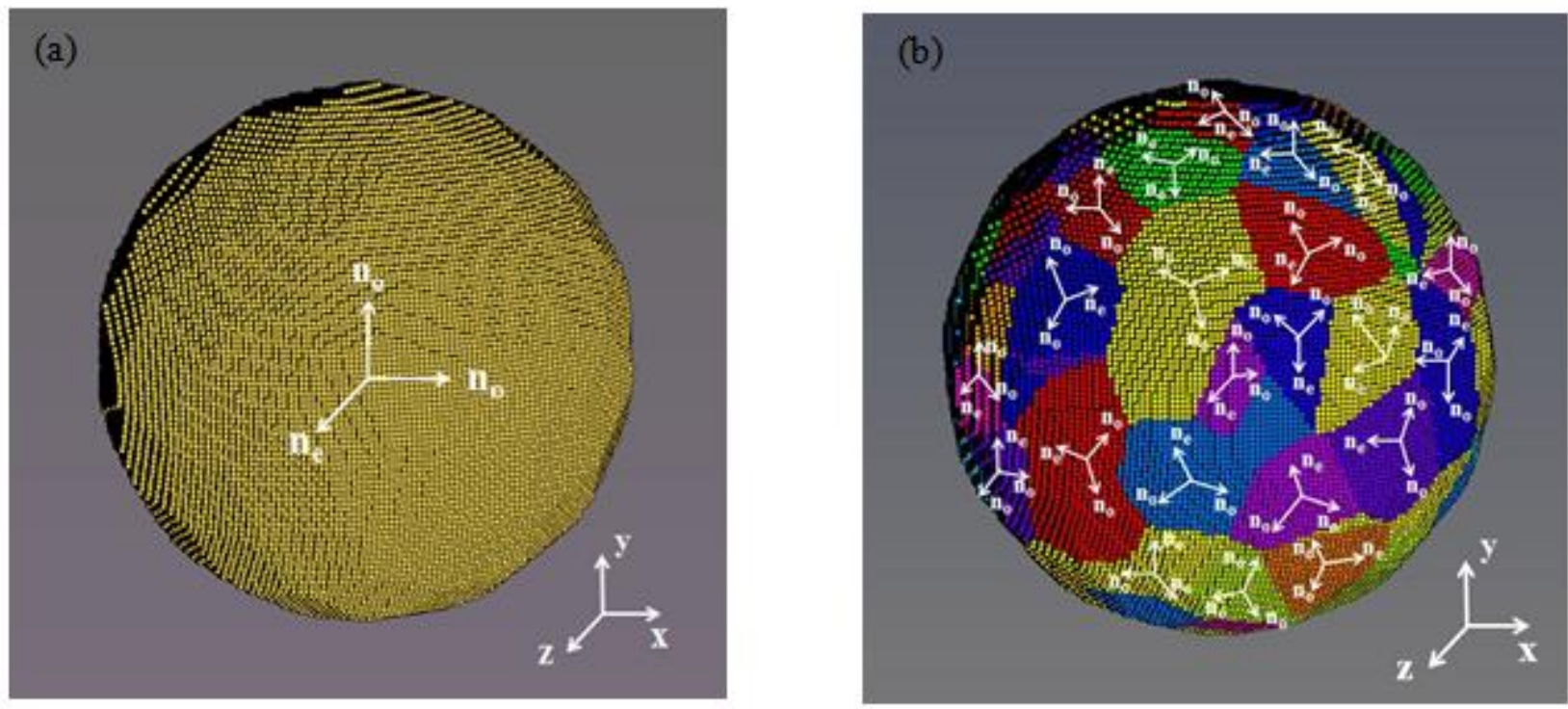

Fig 1. Schematic of BSCS (a) and BPCS with 200 numbers of crystals (b). x, y, z represent the axes of the lab frame. Different crystal domains are represented by different colors. The orientations of the arrows represent the orientation of the index ellipsoids

The birefringent polycrystalline sphere (BPCS) is constructed by grouping the polarizable entities of the sphere, using Voronoi tessellation [13]. Polarizable entities belonging to a Voronoi cell have the polarizable tensor obtained by performing rotational transformation randomly in 3-dimension on the diagonal polarizability tensor. The resulting BPCS would have Voronoi cells representing single crystals oriented randomly as shown in figure 1(b). Linearly polarized light is incident on the particle from $\mathrm{x}$ direction. Scattering cross section as a function of wavelength is calculated with polarization along $y$ as well as z-axis. Three sets of polycrystalline spheres are constructed with 5, 20 and 200 numbers of single crystal domains.

The results obtained are compared to that of an equivalent isotropic sphere (EIS) of the same size, calculated with the refractive index obtained from weighted average of ordinary and extraordinary refractive indices as $m=\frac{2 m_{0}+m_{e}}{3}$ [14].

\section{RESULTS AND DISCUSSION}

Figure 2 shows the scattering efficiency $\left(Q_{s c a}=\frac{c_{s c a}}{\pi a^{2}}\right)$ calculation for EIS, BPCS with 5, 20 and 200 crystal domains and BSCS. Scattering spectrum is calculated for the visible region. Calculation of scattering spectrum of anatase sphere is presented in figure 2(a). For this calculation range of wavelength is chosen such that imaginary refractive index and hence loss due to absorption is completely negligible. Range of the spectrum for rutile sphere in figure 2(b) is chosen such that the mode numbers associated with EIS are same as that observed in the calculation for EIS of anatase phase. In this range of consideration, angular momentum mode numbers observed for TE modes are 3, 4 and 5 and that for TM mode are 3 and 4.
It is observed that in the region of interest for this particular diameter, the observed TE modes are sharper than TM modes for EIS. Higher the order of the mode, sharper is the peak. This trend is followed by EIS of both anatase and rutile phase.

Effect of polarization is clearly visible in the case of BSCS. When light is incident along the axis perpendicular to the optic axis (along x-axis), spectrum due to y-polarization (black curve) is different from spectrum due to $\mathrm{Z}$ polarization (red curve) for both anatase and rutile spheres. When light is incident along optic axis (along z-axis) no effect of polarization is observed for both anatase and rutile spheres. Effect of polarization is more significant in rutile sphere than that in anatase sphere as birefringence of rutile is greater than that of anatase.

Scattering spectra obtained for BPCS are similar to that of EIS with small changes in the region near some of the modes. Though EIS is insensitive to the polarization of incident light, spectra of BPCS show small dependence on polarization of the incident light mostly in the region near the modes. These changes are more significant in rutile than that in anatase. 
(a)
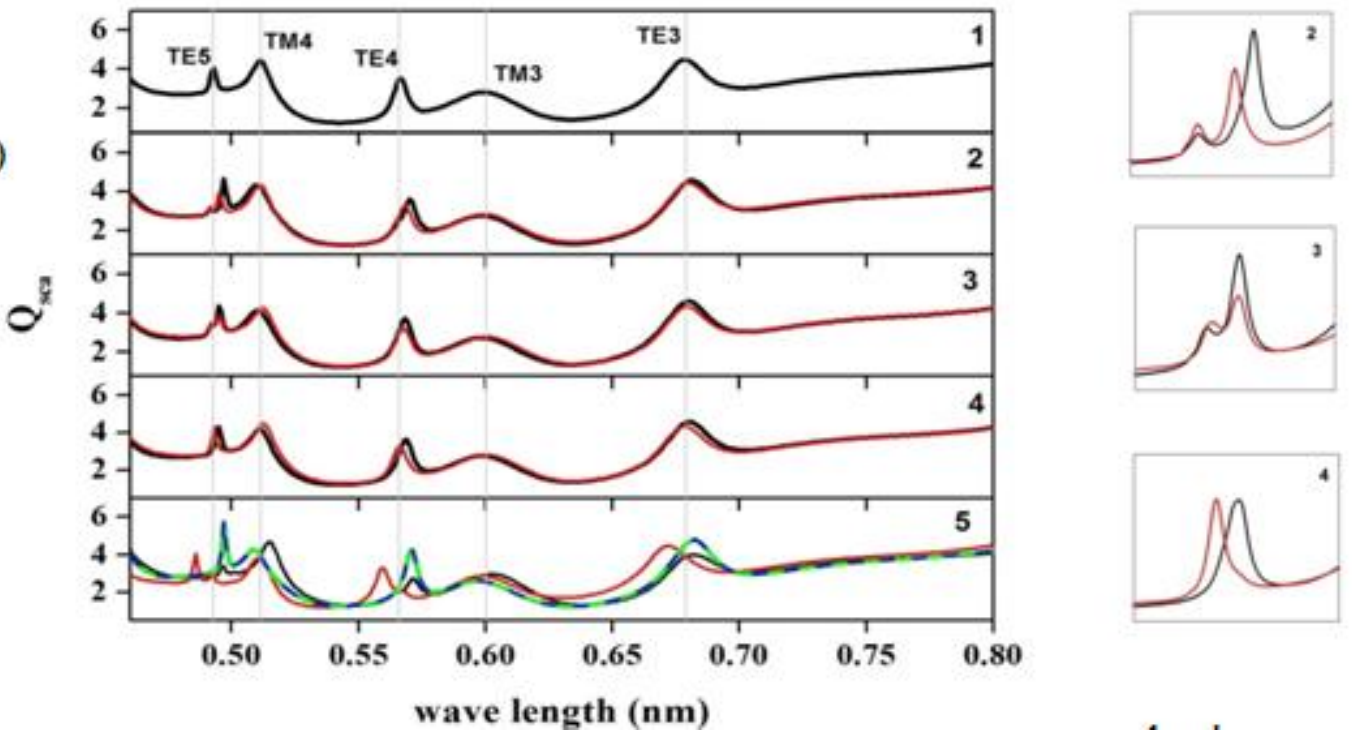

(b)
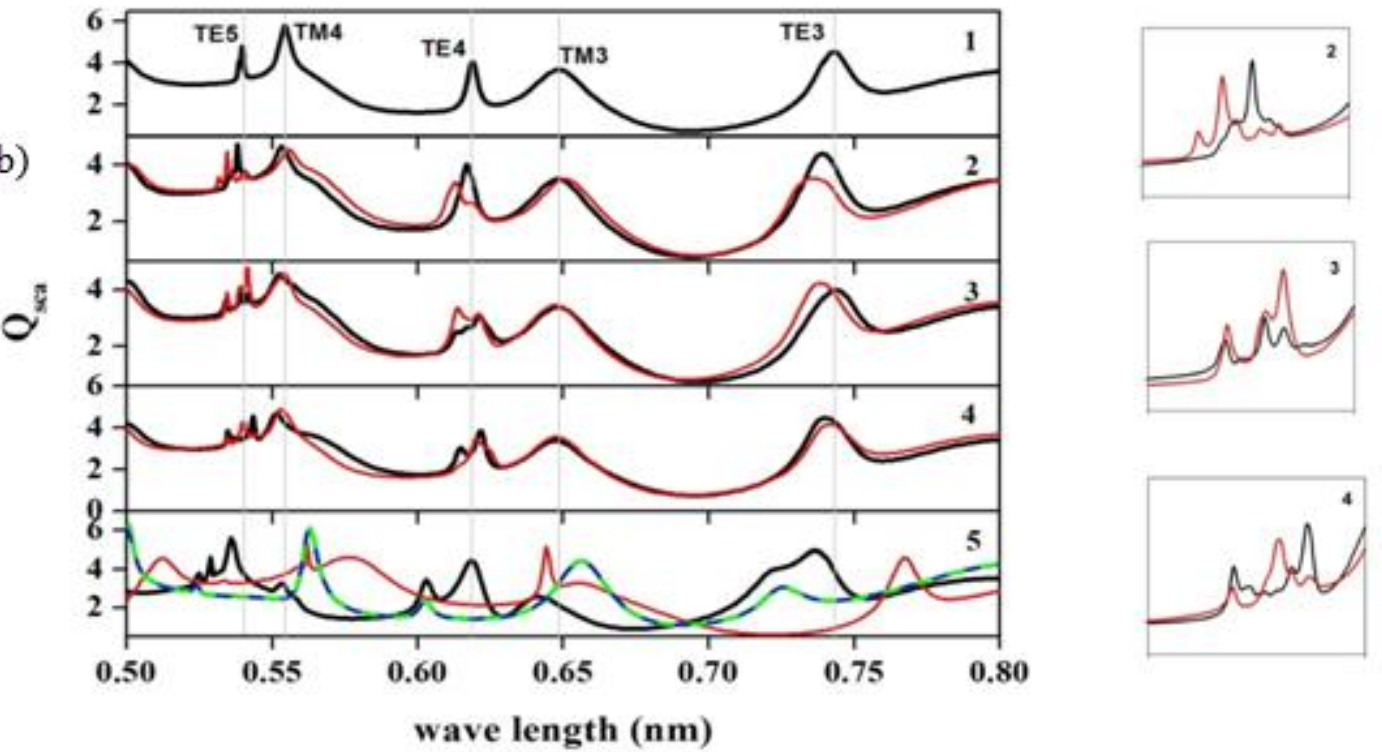

Fig 2. Scattering cross section calculation for (a) anatase (b) rutile spheres of $500 \mathrm{~nm}$ diameter, for EIS (1), BPCS with number of crystallites equal to 5 (2), 20 (3), 200 (4) and BSCS (5). Black and red solid lines represent the case when light incidents along x axis with polarized along $y$ and $\mathrm{z}$ axis respectively and green solid line and blue dashed line represent the cases when light incidents along $\mathrm{z}$ axis and polarized along $\mathrm{x}$ and $\mathrm{y}$ axis respectively. Splitting of $\mathrm{TE}_{5}$ modes for BPCS with number of crystallites equal to 5 (2), 20 (3), 200 (4) are shown in the right side of (a) and (b).

TM modes observed in the case of EIS are also observed in BPCS with little shift and no splitting in both anatase and rutile phases. Significant broadening is observed in the case of $\mathrm{TM}_{4}$ mode of rutile phase. TE modes show significant shift compared to TM modes within the considered range of wavelength. Splitting of modes is observed in the higher order TE modes in both rutile and anatase phases. In the case of anatase sphere, splitting is observed in $\mathrm{TE}_{5}$ mode only. While this splitting is well observed in the BPCS containing 5 and 20 crystals, it is absent in the BPCS containing 200 single crystals. Spread of this splitting is more in the BPCS with 5 single crystals than with 20 single crystals. In the case of rutile sphere, splitting is observed for $\mathrm{TE}_{4}$ and $\mathrm{TE}_{5}$ mode for the BPCS with 5, 20 as well as
200 single crystals. Splitting for $\mathrm{TE}_{5}$ mode is wider with compared to all the other modes under consideration.

\section{CONCLUSION}

From the scattering cross section calculations it has been observed that anisotropy plays a significant role in scattering pattern. Scattering from a single crystalline sphere is completely dependent on the orientation of the sphere with respect to the incident light as well as its polarization. Scattering by such particles cannot be approximated by EIS approximation. In case of polycrystalline spheres TE modes are affected more than TM modes, by polycrystallinity. TE modes of higher orders are highly affected by the 
polycrystalline nature of the sphere. In such particles lower modes can be well approximated by EIS but such approximation may fail to predict the modes of higher orders. In case of polycrystalline materials of high birefringence, EIS approximation is not recommended for estimating the resonance modes. However, for particles made of low birefringence materials and with higher numbers of (or small sized) crystal domains EIS approximation would be suitable for predicting the resonance modes.

\section{ACKNOWLEDGMENT}

We acknowledge HPCE, IIT Madras for high performance computing facility.

E-mail address: * kalyanimon1@gmail.com

+cvkm@iitm.ac.in

\section{REFERENCES}

[1]. R K Chang, A J Campillo, Optical processes in microcavities (World Scientific Publishing Co. Pte. Ltd, London, 1996)

[2]. Matthew R. Foreman, Jon D. Swaim, Frank Vollmer, Advances in Optics and Photonics 7, 168 (2015)

[3]. H.C. van de Hulst, Light scattering by small particles (Dover Publication, Inc., New York, 1981)

[4]. P. J. Flatau, B.T. Draine, Journal of Optical Society of America 11, 1491 (1994)

[5]. P. J. Flatau, B.T. Draine, Optics Express 20, 1247 (2012)

[6]. Erik S. Thiele, Roger H. French, J. Am. Ceram. Soc. 81, 469 (1998)

[7]. Kazuya Nakata, Akira Fujishima, Journal of Photochemistry and Photobiology C: Photochemistry Reviews 13, 169 (2012)

[8]. Yan Hong, Wonmi Ahn, Svetlana V. Boriskina, Xin Zhao, and Bjorn M. Reinhard, J. Phys. Chem. Lett 6, 2056. (2015)

[9]. Andreea Paunoiu, Rakesh Singh Moirangthem and Andreas Erbe, Phys. Status Solidi RRL 9, 241 (2015)

[10]. C. C. Lam, P. T. Leung, and K. Young, Journal of Optical Society of America 9, 1585 (1992)

[11]. E.D. Palik, Hand book of optical constants of solids (Academic press, San Diego, 1998)

[12]. G. E. Jellison, Jr., L. A. Boatner, J. D. Budai, B. S. Jeong, D. P. Norton, Journal of applied physics 93, 9537 (2003)

[13]. R. Quey, P.R. Dawson, F. Barbe, Comput. Methods Appl. Mech. Engrg. 200, 1729 (2011)

[14]. By Erik S. Thiele, Roger H. French, Adv. Mater 10, 1271 (1998) 